\title{
Hoffmann von Fallersleben im aktuellen Fachdiskurs
}

https://doi.org/10.19195/0435-5865.145.24

In den 2000er Jahren lassen sich eine immer größere Geltung und Anziehungskraft Hoffmanns von Fallersleben (weiter: HvF) verzeichnen, sowohl in der breiteren Öffentlichkeit als auch in der Wissenschaft, so dass man besonders in der HvF-Forschung von einer Art Hochkonjunktur sprechen kann. 2014 wurde die neu konzipierte, interaktiv ausgerichtete Dauerausstellung des HvF-Museums im Schloss Fallersleben eröffnet, von der sich - wie man einer Fülle von Rezensionen entnehmen kann - die immer zahlreicher kommenden Besucher angetan zeigen. Diese gelungene Präsentation, die auf das Leben und Werk Hoffmanns auf innovative Art und Weise neugierig macht, wird durch das 2015 mit großer Sorgfalt herausgegebene umfangreiche Begleitbuch zur genannten Ausstellung umrahmt, das einen reichen Fundus der verlässlichen Daten über den Autor des Liedes der Deutschen anbietet. ${ }^{1}$ Neben der attraktiven Ausstellung und dem qualitätsvollen Museumsführer leisten die Aktivitäten der HvF-Gesellschaft mit ihren regelmäßig erscheinenden „Mitteilungen“, in denen originelle Zugänge zu Hoffmann und seinem Werk gefunden werden, einen nicht zu unterschätzenden Beitrag zu seiner Popularisierung.

Ein beredtes Zeugnis für die starke Belebung auf der Forschungsebene ist die Anzahl der wissenschaftlichen Arbeiten aus den letzten beiden Jahrzehnten. Von besonderer Relevanz sind dabei die in der geschätzten Reihe der „Braunschweiger Beiträge zur deutschen Sprache und Literatur" veröffentlichten Studien. ${ }^{2}$ In der 1999 erschienenen Festschrift zum 200. Geburtstag Hoffmanns und in den darauffolgenden Tagungsbänden $(2005,2009,2019)$ wurden die wichtigsten Vertreter der heutigen HvF-Forschung ins Gespräch gebracht und neue unterschiedliche Perspektiven auf das Leben und Werk Hoffmanns eröffnet. Vor diesem Hintergrund soll hier der neueste Forschungsstand zu HvF am Beispiel des 2019 veröffentlichten Bandes August Heinrich Hoffmann von Fallersleben im Kontext des 19. Jahrhunderts und der Moderne betrachtet werden. Es geht um den von Cord-Friedrich Berghahn, Gabriele Henkel und Kurt G.P. Schuster herausgegebenen Tagungsband, der auf das von der HvF-Gesellschaft und dem HvF-Museum in Zusammenarbeit mit dem Germanistischen Institut an der Technischen Universität Braunschweig in der Zeit 15.-17.09. 2017 organisierte Symposion zurückgeht und die damals vorgetragenen 14 Referate in erweiterter Fassung präsentiert.

${ }^{1}$ Bettina Greffrath / Gabriele Henkel / Christin Langermann (Hrsg.) (2015): Hoffmann von Fallersleben. Dichter, Germanist und singender Freiheitskämpfer. Begleitbuch zur Dauerausstellung des Hoffmann-von-Fallersleben-Museums. Hildesheim/Zürich/New York.

${ }^{2}$ Hans-Joachim Behr / Herbert Blume / Eberhard Rose (Hrsg.) (1999): August Heinrich Hoffmann von Fallersleben. Festschrift zum 200. Geburtstag (= Braunschweiger Beiträge zur deutschen Sprache und Literatur, Bd. 1). Bielefeld; Marek Hałub / Kurt G.P. Schuster (Hrsg.) (2005): Hoffmann von Fallersleben. Internationales Symposion Wroctaw/Breslau 2003 (= Braunschweiger Beiträge zur deutschen Sprache und Literatur, Bd. 8). Bielefeld; Norbert Otto Eke / Kurt G.P. Schuster / Günter Tiggesbäumker (Hrsg.) (2009): Hoffmann von Fallersleben. Internationales Symposion Corvey/Höxter 2008 (= Braunschweiger Beiträge zur deutschen Sprache und Literatur, Bd. 11). Bielefeld; Cord-Friedrich Berghahn / Gabriele Henkel / Kurt G.P. Schuster (Hrsg.) (2019): August Heinrich Hoffmann von Fallersleben im Kontext des 19. Jahrhunderts und der Moderne (= Braunschweiger Beiträge zur deutschen Sprache und Literatur, Bd. 18). Bielefeld. 
Die thematische Ausrichtung des Buches wurde eingangs von dessen Herausgebern präzise dargelegt. Sie haben den neuesten Forschungsstand zu HvF erläutert und die im Band veröffentlichten Aufsätze, die ,inhaltlich [...] nach biographischen, werkbezogenen und kunst-resp. musikwissenschaftlichen Aspekten“3 angeordnet wurden, angekündigt.

Den fachlichen Einstieg bieten die ersten drei Aufsätze, die sich biographischen Aspekten widmen, dann spannt sich der Bogen von den auf das dichterische und wissenschaftliche Werk Hoffmanns fokussierten sechs Studien bis zu denen, die sich - so im Vorwort auf S. 11 - den Künsten widmen, vor allem - im abschließenden Teil - der Musik.

Im Falle solcher facettenreichen Tagungsbände kann man die Herausgeber wegen der vorgeschlagenen Reihenfolge der von den Referenten eingesandten Studien „anklagen" und auch in Bezug auf das vorliegende Buch die Frage stellen, ob es nicht sinnvoller wäre, den ertragreichen Aufsatz von Gabriele Henkel „, Mein Bild ist Jahre lang im Bazar ausgestellt gewesen... “. Zu den Hoffmann-Porträts im 19. Jahrhundert, in dem die Lebensperspektivierung stark ersichtlich wird, in den biographischen Themenblock aufzunehmen. Man darf hier das Konzept der Reihenfolge weiter hinterfragen. Aus der Perspektive des Rezensenten wäre es angebracht, Beiträge, die thematisch korrespondieren, aufeinander folgen zu lassen, und den Aufsatz von Gerd Biegel Hoffmanns Verbindungen zur Region mit der Analyse des Heimatmotivs in der Lyrik Hoffmanns aus der Feder Nikolas Immers zusammenzufügen. Auch die Referate von Anett Lütteken und Michael Ewert lassen sich auf einen gemeinsamen Nenner bringen und runden das Themenfeld „Hoffmann als leidenschaftlicher Sammler und Herausgeber“ ab. Im Hinblick auf diese Überlegungen melden sich mit Sicherheit Optionen „Für und wider“ zu Wort, die angesichts dessen, dass der Inhalt dieses Sammelwerkes nicht in Sektionen gegliedert wird, wie auch angesichts des großen wissenschaftlichen Potenzials dieses Bandes lediglich als sekundär erscheinen, geht es in dem hier zu rezensierenden Buch doch primär darum, der neuesten HvF-Forschung Rechnung zu tragen.

Versucht man durch die vielfältigen Beiträge dieses Sammelbandes einen roten Faden zu ziehen, dann gilt es hier zu konstatieren: Die HvF-Forschung ist an einem Punkt angekommen, an dem unser Wissen über HvF punktuell zu ergänzen ist, an dem die mit diversen Details verbundenen Forschungsleerstellen zu füllen sind. So stehen im Mittelpunkt des präsentierten Werkes nicht mehr die bereits ausführlich und vielschichtig besprochenen ,zentralen HvF-Themen“, sei es die viel diskutierte deutsche Nationalhymne, sei es die weit bekannten Kinderlieder oder die bislang mehrmals analysierte politische „Poesie des Grimms“ Hoffmanns und seine Gelehrtenlaufbahn, sondern die bisher kaum oder peripher behandelten Einzelaspekte aus seinem Leben und Werk. Liest man den neu erschienenen Tagungsband aus dieser Sicht, d.h. aus der Forschungsperspektive, dann erkennt man sofort die erhebliche gewinnbringende Erweiterung unseres Wissens über HvF. Angesichts einer Fülle von bemerkenswerten Details, die von den Autoren dieser Publikation in mehreren Kontexten (historischen, soziologischen, philologischen, wissenschafts-, kunst- und mentalitätsgeschichtlichen, aber auch medienkundlichen) beigebracht

3 Cord-Friedrich Berghahn / Gabriele Henkel / Kurt G.P. Schuster (Hrsg.) (2019): August Heinrich Hoffmann von Fallersleben im Kontext des 19. Jahrhunderts und der Moderne (= Braunschweiger Beiträge zur deutschen Sprache und Literatur, Bd. 18). Bielefeld. S. 8 (weitere Seitenangaben im Text). 
werden, konzentriert sich die vorliegende Rezension in Form möglichst kurzer Inhaltsskizzen auf einige Stichworte zu den einzelnen Aufsätzen.

In erster Linie ist hier der Mehrwert bei der werkanalytisch-literaturgeschichtlichen Herangehensweise zu betonen, dank der Themen aus den bislang übersehenen Bereichen aufgegriffen wurden. Von einer besonderen Relevanz sind zwei umfangreiche Studien über das bisher unbeachtete umfassende Quellenkorpus Hoffmanns: Eberhard Rohse nimmt zum ersten Mal Bibel- und Literaturzitate als Gedicht-Motti ,unpolitischer“ Lyrik Hoffmanns unter die Lupe und Kurt G.P. Schuster geht zum ersten Mal auf Hoffmannsche literarische Kleinformen, d.h. Epigramme, Aphorismen und Sprüche ein.

Chapeau bas - möchte man sagen, wenn man sich dem Beitrag Eberhard Rohses widmet, in dem der Verfasser detaillierte Auskunft zu einer Vielzahl von ausgewählten Mottozitaten im facettenreichen Kontext der möglichst breit verstandenen Kulturlandschaft mit besonders starken Bezügen zu Bibel, europäischer und deutscher Geschichte, Literatur und Wissenschaftsgeschichte gibt. Der intertextuelle Ansatz bei der Betrachtung der von Hoffmann verwendeten Motti ist mustergültig ausgefallen und deckt neue Kontextualisierungen bei der Interpretation seiner Gedichte auf.

Chapeau bas - möchte man wiederholen bei der Lektüre des Aufsatzes von Kurt G.P. Schuster. Die von Heinrich Gerstenberg in seiner achtbändigen Edition von Hoffmann's von Fallersleben Gesammelten Werken aus Platzmangel ausgeklammerten unzähligen literarischen Kleinformen werden hier zum ersten Mal in einer breiten Auswahl und hoher Detailfülle wahrgenommen. Es geht um biographisch, politisch, literatur- und kulturgeschichtlich, personenbezogen angelegte Distichen, Xenien und Sprüche aus der Feder Hoffmanns, von denen die von Schuster ausgewählte imponierende Zahl von 100 zitierten Beispielen ein beredtes Zeugnis nicht nur für den faszinierenden Ideenreichtum und Scharfsinn, sondern auch für das äußerst breite Tätigkeits- und Interessenspektrum Hoffmanns ist. Stark hervorzuheben ist in dieser Studie die Ausweitung des bisherigen Blickfeldes auf die Positionierung Hoffmanns in mehreren Lebensbereichen seiner Zeit.

Ein großes Potenzial zeigen auch die Texte von Herbert Blume, Gabriele Henkel und Cord-Friedrich Berghahn; dank ihrer Studien hat das Profil Hoffmanns an Tiefe gewonnen. In einer Vergleichsanalyse wird Hoffmann von Herbert Blume mit dem heute vergessenen, aus dem Kreis Helmstedt stammenden Dichter Eduard Schmelzkopf (18141896) in Verbindung gebracht. Als Demokraten haben beide in ihren Liedern den freiheitlichen Bestrebungen der Vormärzzeit gehuldigt, wobei sie sich in mehrfacher Hinsicht, z.B. im Hinblick auf ihre Anstellung im Staatsdienst, unterschieden haben: Hoffmann war - wie bekannt - im staatlichen Dienst tätig, „Schmelzkopf dagegen entzieht sich dem obrigkeitlichen Wissenschaftsbetrieb der Vormärz-Epoche mit einem Rigorismus, der kaum zu überbieten ist" (S. 73). Solche Vergleichsstudien haben sich bereits mehrfach in der bisherigen HvF-Forschung bewährt; die Konturen von der Persona poetica und Persona practica des Verfassers des Liedes der Deutschen kommen auch hier facettenreich zum Ausdruck, so ähnlich wie in dem im anzuzeigenden Band veröffentlichten Aufsatz von Cord-Friedrich Berghahn u.d.T. ,Besuch auf der Altenburg“ - Hoffmann von Fallersleben und Franz Liszt. Der Verfasser bedient sich „,des methodischen Inventars der Konstellationenforschung" (S. 243) und deckt auf, wie das bereits behandelte Thema „HvF und Weimar“, um hier z. B. auf die von ihm angeführte Arbeit von Irina Kaminiarz August Heinrich Hoffmann von Fallersleben in Weimar 1854 bis 1860, Weimar 1988, zu verweisen, vielfältige Anschlussmöglichkeiten bietet. Nuanciert belegt er, wie das 
Verhältnis zwischen Hoffmann und Liszt, ja, wie sich die Hoffmann-Vertonungen des bekannten Komponisten vor dem Hintergrund der Weimarer Geselligkeit entwickeln. Es sei hier wenigstens auf die abschließende Konstatierung Berghahns hingewiesen: „Laßt mich ruhen, eines der bedeutendsten Lieder im oeuvre Liszts, wäre, wie fast alle seine Lieder, undenkbar ohne die private Verbindung, ja Freundschaft mit Hoffmann von Fallersleben“" (S. 256).

Auch der Aufsatz von Gabriele Henkel weitet mit seiner Gewichtung die vorhandene Blickrichtung der HvF-Forschung aus. Die Autorin konstatiert eingangs mit Recht: „Eine Untersuchung der Hoffmann-Porträts in Malerei und Fotografie steht also bis heute noch aus“ (S. 214), zumal die Zeitgenossen und die Leserschaft „,von kaum einem anderen Dichter seiner Zeit [...] so viele Bilder wie vom Dichter des Liedes des Deutschen" hatten (S. 240). Die Verfasserin nennt die verblüffende Zahl von über 85 HvF-Porträts (S. 213). Vor diesem Hintergrund ist ihre erkenntnisbringende, abbildungsreiche Untersuchung der von ihr ausgewählten 13 Hoffmann-Porträts aus seiner Lebenszeit mit Freude zu begrüßen. Abgesehen von einer Vielzahl interessanter Einzelheiten wird der Leser im erhellenden Beitrag Henkels mit dem Habitus des selbstbewussten, an der öffentlichen Selbstinszenierung stark interessierten, nicht selten auch zur Eitelkeit neigenden Dichters konfrontiert.

Eine nicht zu unterschätzende Bereicherung der Gesamtübersicht zu Hoffmanns Leben und Werk ist dem Musikwissenschaftler Friedhelm Brusniak zu verdanken, der die HvF-Forschung erfolgreich an der Julius-Maximilians-Universität Würzburg etabliert hat. Eine umfangreiche Liste seiner eigenen HvF-Veröffentlichungen wird mit der in diesem Band erschienenen detailreichen Studie über Das Fortleben der Lieder Hoffmanns von Fallersleben in der musikpädagogischen Reformbewegung der Zwanziger Jahre fortgesetzt. Der Autor verweist auf den Wandel des HvF-Bildes, den die pädagogischen Reformmaßnahmen im Bereich des Einsatzes der Hoffmannschen Kinder- und Schullieder in Schulprogrammen zur Folge hatten. Es ist sein großes Verdienst, dass seine Bemühungen um die stärkere Präsenz der musikpädagogischen Perspektivierung in der heutigen HvF-Forschung eine Resonanz in zwei im Entstehen begriffenen Dissertationen finden, über die im Abschlussteil des Bandes berichtet wird. Evi Reißmann, die bei Prof. Brusniak promoviert, stellt ihr Dissertationsvorhaben „August Heinrich Hoffmann von Fallersleben in deutschsprachigen Schulliedersammlungen von 1848 bis 1918 - Studien zur Rezeptions- und Wirkungsgeschichte“ dar. Diesem Arbeitsbericht folgt der letzte Beitrag des Bandes, die Präsentation des Dissertationsvorhabens von Christian Abelein unter der wissenschaftlichen Leitung Friedhelm Brusniaks: „,Ich habe mich so daran gewöhnt, daß ich beinahe nie dichte ohne zugleich zu singen! - Hoffmann von Fallersleben und Hans Michael Schletterer“. Die geplante Dissertation wird auf der Korrespondenz zwischen dem Autor des Liedes der Deutschen und dem Musiker Hans Michael Schletterer basieren und in ihrem Konzept an die Dissertation des Breslauer HvF-Forschers Mariusz Dzieweczyński Im mecklenburgischen Exil. Edition des Briefwechsels zwischen Hoffmann von Fallersleben und seinem Freund Rudolf Müller anknüpfen. Wie Christian Abelein in seinem Bericht einräumt, hat die Doktorarbeit Dzieweczyńskis ,,auch in der Musikforschung für Aufsehen gesorgt und die Frage gestellt, ob hier ein ähnliches Projekt Aufschlüsse über Hoffmanns Musikverständnis geben kann“"(S. 281). Mariusz Dzieweczyński hat nämlich im souveränen Rückgriff auf die handschriftlichen Quellen und mit Materialkenntnis eindrucksvoll die mecklenburgische Zeit Hoffmanns in die HvF-Forschung integriert und 
den vorliegenden Band dank seiner guten Handschriftenkenntnisse mit einem Aufsatz, der das Verhältnis Hoffmanns zu seinem Sohn Franz im Spiegel ihrer Korrespondenz erhellt, eröffnet. Mit diesem Beitrag ist ein junger Wissenschaftler der Breslauer Universität vertreten, an der sich die HvF-Forschung erfolgreich seit 20 Jahren entwickelt. Der vorliegende Band verdeutlicht ein erfreuliches Signum temporis für die aktuellen Bestrebungen auf dem Gebiet der Erschließung von Hoffmanns Leben und Werk, denn er schließt den wissenschaftlichen Nachwuchs mit ein.

Last, but not least sind auch die Studien weiterer Autoren des vorliegenden Bandes wichtige Erträge der 2017 organisierten Tagung. Im Hinblick auf den neuesten Stand der HvF-Forschung runden sie Themenblöcke ab, die die bisherigen Darstellungen und Interpretationen vertiefend zusammenbringen. So zählt Gerd Biegel in seiner biographisch angelegten Zusammenschau relevante Details auf, die Verbindendes zwischen Hoffmann und seiner Geburtsregion sichtbar machen. Heimat-Topoi in der Lyrik des Poeten aus Fallersleben, die bislang hie und da aufgegriffen wurden, werden von Nikolas Immer in einer insgesamt aufschlussreichen Übersicht verfolgt. Mit einer Detailfülle wird von Bernd Zegowitz das Amerika-Bild Hoffmanns, das man bislang u.a. im Rückgriff auf seine Texanischen Lieder behandelte, entworfen. Präsentiert werden auch die äußerst ertragreiche Herausgeber-Tätigkeit Hoffmanns von Anett Lütteken und sein leidenschaftlicher Sammel eifer von Michael Ewert; beide Aufsätze informieren mit einer tiefen Gründlichkeit über die einzelnen Aktivitäten des Fallersleber Dichters. Lütteken betont das breite thematische, „obsessiv recherchierte“ Gebiet des wissenschaftlich interessierten „Anthologen“ Hoffmann (S. 183), Ewert widmet seine Aufmerksamkeit vor allem der für den Sammler Hoffmann leitenden Prämisse der Kulturnation, die dessen editorischen Projekten zugrunde lag.

Es bleibt im Fazit zu wiederholen: Der jüngst erschienene Band ist mit seinen zahlreichen neuen Einzelergebnissen für die HvF-Forschung als ein besonders verdienstvolles Unternehmen anzuerkennen. Das Buch richtet sich sowohl an das akademische Fachpublikum als auch an die interessierte Öffentlichkeit und liefert wichtige Bausteine, die sich zu einem immer vollständigeren HvF-Bild zusammenfügen. Den Umgang mit der Informationsfülle, die der Tagungsband bietet, erleichtert das Personenregister am Ende des Buches, das dank seiner beachtlichen Materialbasis den Blick für HvF schärft und für die weitere intensive Auseinandersetzung mit dem Dichter grundlegend ist.

\section{Literatur}

Behr, Hans-Joachim / Blume, Herbert / Rose, Eberhard (Hrsg.) (1999): August Heinrich Hoffmann von Fallersleben. Festschrift zum 200. Geburtstag. Bielefeld.

Berghahn, Cord-Friedrich / Henkel, Gabriele / Schuster, Kurt G.P. (Hrsg.) (2019): August Heinrich Hoffmann von Fallersleben im Kontext des 19. Jahrhunderts und der Moderne. Bielefeld.

Dzieweczyński, Mariusz (2015): Im mecklenburgischen Exil. Edition des Briefwechsels zwischen Hoffmann von Fallersleben und seinem Freund Rudolf Müller. Bielefeld.

Eke, Norbert Otto / Schuster, Kurt G.P. / Tiggesbäumker, Günter (Hrsg.) (2009): Hoffmann von Fallersleben. Internationales Symposion Corvey/Höxter 2008. Bielefeld.

Greffrath, Bettina / Henkel, Gabriele / Langermann, Christin (Hrsg.) (2015): Hoffmann von Fallersleben. Dichter, Germanist und singender Freiheitskämpfer. Begleitbuch zur Dauerausstellung des Hoffmann-von-Fallersleben-Museums. Hildesheim/Zürich/New York. 
Hałub, Marek (2005): Im schlesischen Mikrokosmos. August Heinrich Hoffmann von Fallersleben. Eine kulturgeschichtliche Studie. Wrocław.

Hałub, Marek / Schuster, Kurt G.P. (Hrsg.) (2005): Hoffmann von Fallersleben. Internationales Symposion Wroctaw/Breslau 2003. Bielefeld.

Ture von zur Mühlen, Bernt (2010): Hoffmann von Fallersleben. Biographie. Göttingen.

Marek Hatub

(Universität Wrocław, Wrocław)

ORCID: 0000-0002-4217-2527

Marek Hałub, Uniwersytet Wrocławski, Instytut Filologii Germańskiej, pl. Nankiera 15, 50-001 Wrocław, Polen, E-Mail: marek.halub@uwr.edu.pl

Received: 27.08.2019, accepted: 8.04.2020

\section{Ein bemerkenswerter Sammelband}

Gábor Kerekes (Hrsg.): Winterlamm. Studien zu Márton Kalász' Roman. Mitteleuropa. Schriftenreihe zur Kultur, Bd 1. Pilisvörösvár: Muravidék Baráti Kör Kulturális, Egyesület 2018, 116 S.*

https://doi.org/10.19195/0435-5865.145.25

Unter dem Titel Mitteleuropa erschien in dem sich intensiv der Kultur der nationalen Minderheiten widmenden Pilisvörösvárer Verlag Muravidék Baráti Kör Kulturális Egyesület der erste Band einer neuen deutschsprachigen Schriftenreihe, die sich - wie man das dem Vorwort entnehmen kann - die Aufgabe gestellt hat, sowohl wissenschaftlich interessierten Lesern als auch Laien Fragen näher zu bringen, die die Kultur und Sprache der Ungarndeutschen betreffen. Der erste Band widmet sich dem Roman Winterlamm von Márton Kalász, der der erste anspruchsvolle Roman war, der 1986 sich mit dem Schicksal der Ungarndeutschen auseinandersetzte. ${ }^{1}$

Die Grundidee, die hinter dem Band steht, war es, jene Studien zu dem Roman von Kalász, die aus der Feder ungarischer Germanistinnen und Germanisten in deutscher Sprache zumeist außerhalb Ungarns im Ausland verstreut in unterschiedlichsten Publikationen bereits erschienen waren und auf diese Weise für einen interessierten Leser nur schwer zugänglich sind, in einer Veröffentlichung zu vereinen. Der Band möchte ausdrücklich ein Hilfsmittel bei der Beschäftigung mit diesem für die Ungarndeutschen bis dato bedeutendsten Roman sein, das zum besseren Verständnis des Werkes beitragen und darüber hinaus etwa auch im Rahmen des Unterrichts zur ungarndeutschen Thematik sowohl an Hochschulen als auch an Gymnasien genutzt werden kann.

Alle Verfasserinnen und Verfasser der Studien stammen aus Ungarn: Anita Czeglédy, Orsolya Erdődy, Gábor Kerekes, Imre Kurdi, Márta Müller, Eszter Propszt und Dezső

* Im Weiteren: WL.

1 Márton Kalász (1986): Téli bárány. Budapest; Deutsch: Márton Kalász (1992): Winterlamm. Graz/Wien/Köln. 